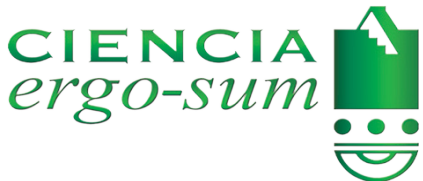

CIENCI A ergo-sum

Universidad Autónoma del Estado de México

ciencia.ergosum@yahoo.com.mx

E-ISSN : 2395-8782

\title{
Oportunidades para la innovación de sistemas tradicionales de producción agropecuaria: un análisis socioantropológico retrospectivo
}

Guevara Hernández, Francisco; Hernández Ramos, Manuel Antonio; Pinto Ruiz, René; Arias Yero, Iliana; Rodríguez

Larramendi, Luis Alfredo; Medina Sansón, Leopoldo y Rodríguez Rodríguez, Sergio

Oportunidades para la innovación de sistemas tradicionales de producción agropecuaria: un análisis socioantropológico retrospectivo

CIENCIA ergo-sum, vol. 26, núm. 1, marzo-junio 2019|e36

Universidad Autónoma del Estado de México, México

Esta obra está bajo una Licencia Creative Commons Atribución-NoComercial-SinDerivar 4.0 Internacional.

Guevara Hernández, F., Hernández Ramos, M. A., Pinto Ruiz, R., Arias Yero, I., Rodríguez Larramendi, L. A., Medina Sansón, L. y Rodríguez Rodríguez, S. (2019). Oportunidades para la innovación de sistemas tradicionales de producción agropecuaria: un análisis soci oantropológico retrospectivo. CIE NCIA ergo-sum, 26(1). https://doi.org/10.30878/ces.v26nla1 


\title{
Oportunidades para la innovación de sistemas tradicionales de producción agropecuaria: un análisis socioantropológico retrospectivo
}

Opportunities for agricultural innovation of traditional farming systems: a retrospective socio-anthropological analysis

\author{
Francisco Guevara Hernández \\ Universidad Autónoma de Chiapas, México \\ francisco.guevara@unach.mx \\ Manuel Antonio Hernández Ramos \\ Universidad de Ciencias y Artes de Chiapas, México \\ tony0886@hotmail.com \\ René Pinto Ruiz \\ Universidad Autónoma de Chiapas, México \\ pinto_ruiz@yahoo.com.mx \\ Iliana Arias Yero \\ Universidad de Granma, Cuba \\ ilianaariasyero@gmail.com \\ Luis Alfredo Rodriguez Larramendi \\ Universidad de Ciencias y Artes de Chiapas, México \\ luislarra2012@gmail.com \\ Leopoldo Medina Sansón \\ Universidad Autónoma de Chiapas, México \\ leomesh@gmail.com \\ Sergio Rodriguez Rodriguez \\ Universidad de Granma, Cuba \\ srodriguezr@udg.co.cu
}

Recepción: 26 de abril de 2017

Aprobación: 07 de febrero de 2018

\section{Resumen}

Se identifican los principales cambios históricos (1984-2015) en el manejo de los sistemas productivos de maíz (Zea mays L.), frijol (Phaseolus vulgaris L.) y ganadería bovina (Bos spp.) en la comunidad Francisco Villa I, aledaña a la Reserva de la Biosfera "La Sepultura" (REBISE), Chiapas, México, para encontrar puntos de entrada (oportunidades) a procesos de innovación local. Se utilizó el enfoque socioantropológico y de procesos de innovación social con la metodología de la investigación-acción. Mediante el análisis de discurso y los gráficos de tendencia de las variables estudiadas, se demostró que, con base en el análisis de cambios históricos, existen condiciones locales para emprender nuevos procesos de innovación social que pueden mejorar el desempeño de los sistemas de producción agropecuaria.

Palabras clave: sistemas productivos, manejo agronómico, innovación local, comunidad.

\section{Abstract}

This article identifies main historical changes (1984-2015) in three farming systems: maize (Zea mays L.), beans (Phaseolus vulgaris L.), and cattle (Bos spp.) in "Francisco Villa I" community, located in the surrounding area of the Biosphere Reserve "La Sepultura" 
(REBISE), Chiapas, Mexico. It was conducted in order to find out entry points for subsequent local innovation processes. A socioanthropological and social innovation approaches were used and an action research methodology for the field work was implemented. Discourse analysis and systemic graphics allowed analyzing all the field evidences. It was demonstrated that through the historical changes analysis there are local conditions to enable and undertake new social innovation processes in order to enhance the community farming systems performance.

KEYWORDS: farming systems, agronomic management, local innovation, community.

\section{INTRODUCCIÓN}

La innovación es el resultado de los conocimientos que generan hombres y mujeres tanto de centros de investigación, universidades, como de comunidades. Surgen las innovaciones cuando la ciencia, la tecnología y la sabiduría popular han limitado su eficacia y eficiencia y/o cuando las condiciones objetivas, sean de naturaleza interna o externa, lo indiquen. En el caso de la innovación agropecuaria es el momento preciso para sustituir métodos obsoletos por otros alternativos que mejoren los indicadores de producción en determinado territorio, región o nación. Los sistemas agropecuarios han garantizado su sostenibilidad en el tiempo debido a la incorporación de nuevas prácticas y tecnologías, lo cual es resultado de la creación y recreación de los saberes de los productores, difundidos por tradición oral y medios escritos que describen los cambios entre escenarios y generaciones. Es así que la innovación, como proceso de transición, trasciende de lo individual a lo social (Röling, 1996; Salomón y Engel, 1999; Guevara y Rodríguez, 2011; Knickel et al., 2009).

Por lo anterior, el objetivo fue analizar el desarrollo histórico del manejo de los sistemas productivos de maíz (Zea mays L.), frijol (Phaseolus vulgaris L.) y ganadería bovina (Bos spp.) en la comunidad Francisco Villa I para encontrar puntos de entrada a procesos de innovación local en función de los sistemas locales existentes y, por ende, contribuir al mejoramiento productivo a partir de la investigación-acción y la capacitación de productores in situ. Cumplir con tal propósito significaría para la comunidad Francisco Villa I, el descubrimiento de la puerta de entrada a la innovación en estos sistemas, los cuales han formado históricamente parte de la idiosincrasia productiva de la localidad y su perfeccionamiento constituye un eslabón en la cadena del desarrollo como sistema complejo; sin embargo, para emprender procesos de innovación agropecuaria las comunidades rurales, en general, poseen recursos limitados y condiciones adversas que obstaculizan su desarrollo, como lo indican Mosse (2005) y Uphoff (1986).

\section{LA INNOVACIÓN COMO PROCESO SOCIAL}

La innovación ha sido analizada y comprendida desde diferentes perspectivas y se ha entendido como a) un proceso de modificaciones, $b$ ) sistema de información que tiene utilidad práctica, $c$ ) mecanismo que apoya la difusión de conocimientos y tecnologías, d) productos acabados y e) proceso de aprendizaje individual o colectivo, entre otros (Rogers, 1983; Salomón y Engel, 1999). Sin embargo, para entender la innovación en un sentido académico, y como resultado de un proceso humano más allá del de la investigación, es importante definirla sobre la base de algunos elementos clave como los siguientes:

a) Innovar significa estar con lo nuevo, es decir, en constante cambio. Es una aventura hacia lo desconocido o imprevisible.

b) Es una idea que se lleva a la práctica, que busca mejorar lo ya realizado, hacerlo de manera diferente o más eficiente.

c) Es un producto nuevo que no se había hecho.

d) Es algo que busca ahorrar esfuerzos, ser útil y satisfacer necesidades. 
e) Debe producir beneficios como la reducción de tiempo, esfuerzo o espacio, etcétera.

f) Significa sumar y transformar energías. Es decir, es un esfuerzo coordinado de actores donde se aplica y transforma la realidad para obtener un beneficio.

g) Debe ser sencilla, compatible, para que se utilice por mucha gente.

b) Intenta ser ambientalmente amigable.

i) Debe generar o aumentar ganancias, esto es, ser rentable e impactar positivamente en el crecimiento económico (Santiago, 1985).

No obstante, la innovación, vista como un proceso intelectual que surge desde la capacidad humana de imaginar, racionalizar y socializar, supone una lógica dialéctica que construye una idea y se entrelaza con varias de ellas que se llevan a la práctica, pues la innovación lo es en sí cuando logra alcanzar la praxis social con el objetivo de generar cambios para satisfacer una necesidad o facilitar alguna actividad productiva del hombre. Si bien esta acepción coincide con el criterio de un autor clásico como Rogers (1983), discrepa en cuanto a que el carácter individual o colectivo del proceso de aprendizaje no es alternativo, pues si se limita a una persona, simplemente no es innovación colectiva, ya que se niega la perspectiva social del mismo y la práctica como requisito sine qua non para ratificar su esencia (Salomón y Engel, 1999; Rist et al. 2006).

Los estudios realizados por Arocena y Sutz (2000), Lundvall y Johnson (1994) y Partida (1996) en innovación industrial aclaran que la innovación incluye la intermediación de la sociedad a través de los procesos de búsqueda de sus satisfactores. Por lo tanto, al verla como proceso, tiene consecuencias de cambio de tipo técnico, socioeconómico, político, ambiental, cultural, los cuales a su vez son factores determinantes (Johnson y Andersen, 2012; Méndez Gutiérrez del Valle, 2010; Young, 2009).

Por su parte, Represas (1962), en su libro Ciencia y Tecnología en la Industria, ya desde los años sesenta se refería a algunos ejemplos clave para entender la innovación; es el caso del desarrollo de los ferrocarriles y su aportación al desarrollo de la civilización. Este suceso fue propiciado por los progresos en la técnica para producir hierro y más tarde acero (Corona y Hernández, 2001; Méndez Gutiérrez del Valle, 2010).

Arias et al. (2008) mencionan que la innovación social es la transformación que introduce cambios cualitativos en la forma de organizar algunos de los modelos de activación social, por ejemplo en la calidad de vida y que son resultado de cambios endógenos o por la acción de agentes externos. Dichos modelos son una forma de manejo organizacional que fomenta la innovación por medio de la toma de decisiones colectivas de una manera organizada y funcional. En este sentido, Rodríguez y Alvarado (2008) indican que la innovación es un proceso endógeno, donde los resultados se atribuyen a causas internas, de manera propia, pero también es exógeno porque los resultados se producen a partir de la intervención de factores externos. Por otra parte, Salazar y Rosabal (2007) mencionan que es un proceso social emergente de procesos múltiples con capacidad para crear nuevos o diferentes procesos. Por el contrario, Salomón y Engel (1999) indican que es un proceso social que comprende la generación y aplicación del conocimiento como una construcción social, donde participan actores clave para afrontar los desafíos del desarrollo.

De acuerdo con Guevara y Rodríguez (2011), la innovación es un proceso de aprendizaje, donde conocimientos individuales o colectivos previos se combinan para llevar a la práctica las ideas que motivan a desarrollar cambios en alguna tecnología, organización o cadena productiva. Al respecto, Bellón (2002) clasifica el conocimiento de los agricultores en tres categorías: a) las percepciones, las cuales son imágenes mentales que se producen por la medición de los sentidos, $b$ ) las taxonomías que representan la abstracción de las percepciones y la clasificación en sus categorías con nombres y propiedades definidas, clasificadas en forma jerárquica con alto grado de aceptación dentro de una población dada y c) reglas empíricas, las cuales son proposiciones lógicas que asocian dos acontecimientos en una relación de causa-efecto.

Por tanto, la innovación vista como proceso representa que se generen métodos de producción nuevos o significativamente mejores que puedan involucrar al equipo, a la organización o a ambos. Los métodos 
pueden enfocarse en crear nuevos productos que no se logran de forma tradicional, así como a mejorar la eficiencia productiva de los ya existentes (Muñoz et al., 2004).

\section{LA INNOVACIÓN AGROPECUARIA Y LAS CAPACIDADES DE LOS SISTEMAS PRODUCTIVOS}

La innovación en la agricultura, en su sentido más amplio, es vista como el resultado final de un proceso humano que tiene dos puntos de partida, pero que pueden presentarse de manera paralela, simultánea, independiente o complementaria. El primero es la experimentación científica, donde los ensayos establecidos formalmente como parte de la investigación básica aplicada o adaptativa buscan instaurar los cambios o las mejorías en los sistemas productivos. El segundo parte del descubrimiento, uso, adopción o adaptación de nuevas técnicas por parte de los productores y se origina mediante pruebas de ensayo y error, resultado de lo que se conoce como experimentación campesina (Guevara Hernández et al. 2008).

En este sentido, los agricultores llevan a cabo distintos y variados experimentos como parte de su proceso de innovación (Scoones y Thompson, 1994; Rhodes y Bebbington, 1988). Entre las pruebas que realizan los agricultores se pueden citar: $a$ ) experimentos por curiosidad sólo para ver qué pasa, $b$ ) experimentos para solucionar problemas específicos, $c$ ) experimentos de adaptación en los que se adecuan tecnologías nuevas a ambientes conocidos o tecnologías conocidas a ambientes nuevos y $d$ ) experimentos fortuitos, es decir, acontecimientos imprevistos que originan cambios en la práctica y que a su vez crean nuevas experiencias de aprendizaje. Estos autores refieren criterios más abarcadores clasificándolos en tipos de experimentos que responden bien a las causas capaces de generar la innovación en los sistemas productivos.

El conocimiento de los agricultores es dinámico, pues además de sus propios saberes, asimilan o rechazan información, datos y conceptos que reciben de los servicios de extensión, escuelas de agronomía, proveedores de insumos y servicios agropecuarios, medios de comunicación y otras fuentes (Bellón, 2002). Entonces, para estimular los procesos de innovación es pertinente que los productores estén motivados y dispuestos a realizar el cambio pues, aunque la ganancia resulte evidente, la resistencia a las transformaciones puede convertirse en una limitante.

La innovación agropecuaria parte de una necesidad y su primer objetivo es precisamente satisfacerla, pero si la tecnología actual produce resultados óptimos y los indicadores productivos cubren las expectativas alimenticias de exportación y de ingresos directos al productor, entonces la pregunta sería ¿para qué innovar? La decisión entre innovar o no, y para qué, se relaciona con el balance entre las necesidades actuales y los satisfactores de bienes de uso directo o indirecto disponibles y producidos a partir de la innovación. Sin embargo, los satisfactores se ubican en tiempos y espacios dinámicos que delimitan la temporalidad de una innovación (Guevara y Rodríguez, 2011).

En otra línea de entendimiento, algunos autores sugieren que para emprender procesos innovadores se deben priorizar aquellos elementos que signifiquen mayor impacto económico, aumento de la calidad de vida y desarrollo de la comunidad y que además generen beneficios, por ejemplo, la satisfacción de necesidades alimenticias en coherencia con las tradiciones culinarias de la región (Knickel, 2009; Toledo et al., 1985; Wenger et al., 2002)

En congruencia con Santiago (1985), la comunidad y sus productores deben poseer habilidades para difundir el conocimiento innovador en todas las direcciones, ya que según Rogers (1983) y Waitley y Tucker (1989) esto constituye una tercera etapa como proceso que impulsa el desarrollo de la sociedad, después de la invención y la innovación propiamente.

En síntesis, revisar el trabajo de varios autores permite interpretar la innovación agropecuaria como un proceso social complejo que obedece a disímiles circunstancias con ciertas condicionantes para emprenderlo y encontrar los satisfactores que cubran las necesidades objetivas como alimentación, adaptabilidad a los cambios del sistema, disponibilidad de los productores para la transición y, por consiguiente, visualizar la 
posibilidad de generar impactos económicos significativos que se traduzcan en mejores condiciones de vida para la comunidad.

\section{Metodología}

\section{1. Área de estudio}

El estudio se desarrolló durante 2014 y 2015 en la comunidad Francisco Villa I, municipio de Jiquipilas, Chiapas, México, dentro del área de influencia de la Reserva de la Biosfera "La Sepultura” (REBISE), que se localiza al sureste del estado, sobre la Sierra Madre de Chiapas. La comunidad presenta topografía accidentada y planadas en las zonas más bajas, con vegetación primaria característica de selva baja caducifolia como las especies de Bursera spp., Leucaena spp., Gliricidia sepium, Byrsonima crassifolia y pinos (Pinus chiapensis) y clima de trópico seco. Esta comunidad se constituyó en ejido en 1984 con 32 ejidatarios. [1] Actualmente cuenta con 40 ejidatarios y 55 pobladores. Los sistemas de producción agropecuaria son tradicionales, con ciertos cambios tecnológicos incorporados en los últimos años.

\section{2. Tipo de investigación}

Esta investigación es del tipo cualitativa y tiene su base teórica en los postulados de Rogers (1983), Salomón y Engel (1999) y Waitley y Tucker (1989) respecto al estudio de la innovación como proceso social que involucra un aprendizaje individual y colectivo. Además, se centra en la acción humana sobre su contexto, donde el individuo como ente se observa bajo la mirada de su tejido social, su forma de organización y de construir instituciones para dar respuesta a las necesidades sociales; en este caso es la producción de alimentos, de acuerdo con Giddens (1982).

Para obtener la información se emplearon métodos y herramientas que documentaron acciones locales en el marco de la dinámica cotidiana del desarrollo agroproductivo de la comunidad (Hernández, 1980). Se utilizó una metodología de investigación-acción en la que se aplicaron métodos participativos que estimularon la reflexión con los productores sobre el tema abordado (Almekinders et al., 2009; Keune y Boog, 2000; Selener, 1997). Es decir, el trabajo de campo involucró gradualmente a los productores agropecuarios de la comunidad para profundizar con ellos en el análisis de los procesos locales de innovación agropecuaria a partir del respeto a su dinámica interna y de sus formas de organización social (Guevara et al., 2009; Guevara, 2007). Para esto, se desarrollaron talleres participativos, entrevistas semiestructuradas, pláticas informales, observaciones directas y recorridos por las parcelas.

\section{3. Compilación y análisis de información}

La colecta de información de campo procedió de la siguiente manera:

a) Contacto con autoridades de la comunidad para obtener su autorización e invitar a los productores a participar en el estudio.

b) Diagnóstico de la comunidad: población, recursos naturales disponibles, sistemas productivos, etcétera.

c) Talleres comunitarios mediante invitación abierta. En tres talleres participaron 71 jefes de familia de los 95 existentes en la comunidad. La primera sesión estuvo dedicada a sensibilizar a los productores en lo referente a la investigación para garantizar la validez de la colectiva de la información vertida durante los eventos. En la segunda se analizó la situación actual de sus sistemas productivos y en la tercera se detectaron las perspectivas de los productores en relación con los posibles cambios por 
implementar en sus sistemas agropecuarios. Todas las sesiones tuvieron carácter participativo donde fue evidente la reflexión y el análisis profundo con los asistentes. En los talleres se identificaron elementos que describen los cambios históricos en los sistemas de producción agropecuaria y con la información obtenida se integró en una línea de tiempo que fue analizada e interpretada etnográficamente.

d) Identificar a los informantes clave para su entrevista con el fin de obtener apuntes sobre tópicos específicos después de cada taller, sobre todo para triangular información que fuera necesario verificar posteriormente, de acuerdo con Guevara (2007). Los informantes clave en las entrevistas semiestructuradas y pláticas informales fueron lo más diverso posible; para esto, se consideró equidad de género y rol en la comunidad (autoridades ejidales y personas sin cargo alguno en las estructuras comunitarias).

e) Aplicación de entrevistas semiestructuradas a una muestra de 50 productores, jefes de familia, para recolectar información adicional útil al momento de identificar cambios que han realizado en los sistemas agropecuarios, además de corroborar o complementar los datos obtenidos en los talleres.

A continuación, las variables documentadas de los cambios que se implementaron en los sistemas de producción de maíz, frijol y ganadería bovina:

a) Tiempo de practicar los sistemas de producción

b) Cambios realizados en los sistemas de producción

c) Fuente/razón que generó ese cambio

d) Impacto de los cambios realizados

e) Importancia de realizar cambios en los sistemas de producción

f) Necesidades para realizar los cambios en los sistemas de producción

g) Perspectiva de los sistemas de producción

Con los valores asignados por los productores a los cambios experimentados en sus sistemas productivos, en una escala del 0 al 5, donde 0 corresponde al valor mínimo y 5 representa el valor máximo, se elaboró una base de datos y también gráficos de tendencia conocidas como amibas o arañas, como lo sugieren Almekinders y Guevara (2008) y Guevara (2007). Dichos valores fueron de gran utilidad para analizar e interpretar la evolución, tendencia o retroceso de los cambios, así como para identificar "puntos de entrada" a la innovación social sobre la base de los sistemas agroproductivos locales. Cuando fue factible se logró estimar los porcentajes de algunas variables que son presentadas como tal en los resultados.

\section{Resultados y discusión}

\section{1. Descripción evolutiva y actual de los sistemas de producción}

\section{1. 1. Sistema de producción de maiz}

En la tabla 1 y figura 1 se observan los cambios y las actividades que han caracterizado el sistema de producción de maíz desde una línea base de su evolución hasta las prácticas de manejo que aún prevalecen, como parte de la dinámica de dicho sistema.

Entre abril y mayo, la primera actividad agrícola que realiza el productor para el caso del maíz consiste en la eliminación de residuos de la cosecha anterior y las arvenses, conocidas localmente como malezas. A esta práctica se le conoce como rastrojeo. La quema de tales residuos orgánicos se efectúa en mayo. 
TABLA 1

Innovaciones en el sistema de producción de maíz en la comunidad Francisco Villa I (1984-2015)

\begin{tabular}{|c|c|c|c|}
\hline \multirow{2}{*}{$\begin{array}{l}\text { Componente } \\
\text { tecnológico }\end{array}$} & \multicolumn{3}{|c|}{ Periodo/cambio } \\
\hline & $\begin{array}{c}\text { Periodo I } \\
1984-1990\end{array}$ & $\begin{array}{l}\text { Periodo II } \\
1991-2000\end{array}$ & $\begin{array}{l}\text { Periodo III } \\
2001-2015\end{array}$ \\
\hline $\begin{array}{l}\text { Preparación del } \\
\text { suelo y control de } \\
\text { arvenses (malezas) }\end{array}$ & R-T-Q[2] & $\begin{array}{l}\text { Inicia el uso de herbicidas y } \\
\text { manejo controlado del fuego }\end{array}$ & $\begin{array}{l}\text { Continúa el uso de herbicidas } \\
\text { y manejo controlado del } \\
\text { fuego }\end{array}$ \\
\hline Fertilización & $\begin{array}{l}\text { Se generalizó el uso de guano } \\
\text { (abono natural creado a partir } \\
\text { de excrementos de aves y } \\
\text { murciélagos), traído desde } \\
\text { Arriaga, Chiapas }\end{array}$ & $\begin{array}{l}\text { Comienza el uso de } \\
\text { fertilizantes químicos }\end{array}$ & $\begin{array}{l}\text { Continúa el uso de } \\
\text { fertilizantes químicos y en } \\
2008 \text { inician las pruebas con } \\
\text { abonos verdes }\end{array}$ \\
\hline $\begin{array}{l}\text { Decreto de la } \\
\text { REBISE }\end{array}$ & $\begin{array}{l}\text { Manejo tradicional de los } \\
\text { recursos naturales de acuerdo } \\
\text { con usos y costumbres } \\
\text { locales }\end{array}$ & $\begin{array}{l}\text { En } 1994 \text { se decreta la } \\
\text { Reserva de la Biósfera "La } \\
\text { Sepultura". El ejido queda } \\
\text { dentro del área aledaña }\end{array}$ & $\begin{array}{l}\text { Programa de manejo de } \\
\text { agricultura sedentaria }\end{array}$ \\
\hline $\begin{array}{l}\text { Prácticas de limpia } \\
\text { del cultivo para } \\
\text { control de arvenses }\end{array}$ & Manual, con coa & $\begin{array}{l}\text { Uso de herbicidas } \\
\text { Quemas controladas }\end{array}$ & $\begin{array}{l}\text { Uso de herbicidas } \\
\text { Quemas controladas }\end{array}$ \\
\hline $\begin{array}{l}\text { Aplicación de abono } \\
\text { de corral }\end{array}$ & Ningún abono aplicado & Ningún abono aplicado & $\begin{array}{l}\text { En } 2006 \text { una persona adopta } \\
\text { esta práctica, también } \\
\text { conocida como aplicación de } \\
\text { majaleo y en } 201560 \% \text { de } \\
\text { los productores lo adopta }\end{array}$ \\
\hline Tipo de semillas & $\begin{array}{l}\text { Se usaban maíces como } \\
\text { H-501, H-507 y criollos }\end{array}$ & $\begin{array}{l}\text { Empiezan a sembrarse } \\
\text { híbridos como los de la línea } \\
\text { Pionner }{ }^{\circledR} \text {. }\end{array}$ & $\begin{array}{l}\text { Entran programas de apoyo } \\
\text { de maíces mejorados gracias } \\
\text { al municipio }\end{array}$ \\
\hline $\begin{array}{l}\text { Capacitación en } \\
\text { manejo de prácticas } \\
\text { alternativas }\end{array}$ & $\begin{array}{l}\text { Ninguna capacitación } \\
\text { recibida }\end{array}$ & $\begin{array}{l}\text { Días demostrativos para uso } \\
\text { de herbicidas }\end{array}$ & $\begin{array}{l}\text { Módulos demostrativos } \\
\text { para estabelecimiento de } \\
\text { ensilados de maíz }\end{array}$ \\
\hline $\begin{array}{l}\text { Destino de la } \\
\text { producción }\end{array}$ & Autoconsumo & $\begin{array}{l}\text { Autoconsumo y } \\
\text { alimentación animal }\end{array}$ & $\begin{array}{l}\text { Autoconsumo y } \\
\text { alimentación animal }\end{array}$ \\
\hline
\end{tabular}

Fuente: elaboración propia.

\begin{tabular}{|l|l|l|l|l|l|l|l|l|l|l|l|l|}
\hline Actividad/Mes & Ene & Feb & Mar & Abr & May & Jun & Jul & Ago & Sep & Oct & Nov & Dic \\
\hline Rastrojeo/control de malezas & & & & & & & & & & & & \\
\hline Tirado de abono de corral & & & & & & & & & & & & \\
\hline Rastreo & & & & & & & & & & & & \\
\hline Siembra & & & & & & & & & & & & \\
\hline Limpia del cultivo & & & & & & & & & & & & \\
\hline Fertilización & & & & & & & & & & & & \\
\hline Control de plagas & & & & & & & & & & & & \\
\hline Cosecha & & & & & & & & & & & \\
\hline
\end{tabular}

FIGURA 1

Calendarización de actividades del sistema de producción de maíz de temporal en la comunidad Francisco Villa I 
En el análisis retrospectivo de las actividades en este sistema de producción se observa que durante el periodo comprendido entre 1984 y 1990, los productores realizaron desmontes con la práctica de rozatumba-quema (R-T-Q). Luego del decreto de la REBISE (1994) este sistema se ha regulado a través de las quemas controladas debido a los efectos perjudiciales que ejercen al ecosistema.

En 1990 comienza el uso del control químico de malezas y se lleva a cabo una vez que ocurren las primeras lluvias del temporal, lo cual culturalmente se conoce como inicio del ciclo agrícola (por lo general es a fines de mayo) cuando emergen las primeras malezas, que son controladas con deshierbes manuales o aplicación de herbicidas (figura 1). Posteriormente se efectúa la siembra, que desde 1984 se ha llevado a cabo a finales de mayo y principios de junio. En la actualidad se siembra cuatro o cinco días después del control químico de las malezas. En las tierras bajas es mecanizada y en las laderas se realiza con macanas (espeque); [3] es decir, el uso de la maquinaria agrícola depende de la topografía del terreno.

Cuarenta días después de la siembra del maíz, se hace un control químico con Esterón ${ }^{\circledR}$ fundamentalmente o mediante el deshierbe manual, para luego fertilizar. Esta práctica agrícola comenzó entre 1991 y 2000.

En 1984 se sembraban algunas variedades de maíz mejoradas, conocidas como H-501 y H-507. También se producían de tipo criollos como el jarocho, el elotillo o el maíz crema y el higuera, un tipo de maíz blanco. Después se usaron variedades mejoradas de la línea Pioneer ${ }^{\circledR}$, las cuales pusieron en riesgo la existencia de los maíces nativos conocidos localmente como "criollos". En tal sentido, Aurelio "X", un productor de 72 años, menciona: "Con la aparición de los maíces híbridos, los criollos se fueron acabando. En el año 1994 sembré maíz tuxpeño". Por su parte, Nazario "N", otro productor de 70 años añade: "Ese año saqué tres toneladas de seis hectáreas debido a que no llovió. El año anterior se obtuvo un promedio de dos toneladas por hectárea usando semillas Pioneer ${ }^{\circledR}$, el cual es un híbrido blanco. Hasta ahorita sigo utilizando Pioneer ${ }^{\circledR}$, el cual en años buenos, como el 2014, ha rendido hasta cuatro toneladas por hectárea”.

Entre los cambios más significativos añadidos al sistema de producción de maíz, enmarcados en el periodo 1984-1990, esta la fertilización, práctica que los productores realizan durante junio y julio. En este periodo, era básicamente orgánica, a base de guano de murciélago, con dosis que oscilaban entre 200 a $250 \mathrm{~kg} \mathrm{ha}^{-1}$. En la actualidad, la innovación combina factores como $a$ ) tiempo de fertilización, $b$ ) tipo y $c$ ) dosis de fertilizantes aplicados. La primera aplicación es a los siete días de germinada la semilla, aunque el periodo difiere de acuerdo con el productor. Jeremías "C", de 43 años por ejemplo, coincide con esta propuesta, mientras que Nazario "N" prefiere esperar 15 días. En cuanto al tipo de fertilizante, en las primeras aplicaciones se usa sulfato de amonio, llamado también por los productores sal con chile o con nitrato de amonio a dosis de 33, 5-00-00 y fosfato de amonio, llamado también negrito, a razón de 18-46-00.

La segunda aplicación de fertilizante se realiza a los 45 días posteriores a la germinación, con nitrato de amonio o urea y sulfato de amonio. Las dosis de aplicación son de $400 \mathrm{~kg}$ de nitrato de amonio y de $100 \mathrm{~kg}$ de sulfato de amonio, ambas por hectárea, fraccionadas las dos a razón de $200 \mathrm{~kg}$ en la primera y $300 \mathrm{~kg}$ en la segunda aplicación.

Resulta interesante corroborar la inclusión de prácticas innovadoras en el sistema de fertilización del cultivo del maíz. Alexer "F", de 52 años, menciona que con el tirado de abono de corral o majaleo, ${ }^{[4]}$ actividad que se realiza en mayo, ha obtenido resultados positivos desde 2006 con lo que se ha logrado recuperar el suelo en una parcela de 0.5 ha. Al comprar una parcela degradada por mal uso de la tierra decidió aplicar estiércol de ganado bovino, a razón de 5 toneladas en el primer año y 8 toneladas en el segundo. Actualmente, 60\% de los productores de maíz aplica una tonelada menos que la porción inicial. Posterior a la fertilización se realiza el rastreo con tractor en junio para preparar el suelo y mezclarlo con el abono. Por último, la cosecha del maíz se lleva a cabo en diciembre.

En resumen, en la comunidad de estudio el sistema de producción de maíz evolucionó en varios sentidos. De 1984 a 1995 la superficie sembrada por productor pasó de 15 a 20 ha, lo cual no resulta tan relevante debido a que en 11 años sólo incrementó en 5 ha. El rendimiento creció de 3 a 4 t ha-1. Sin embargo, de 1994 a 2015 la superficie sembrada ha disminuido aproximadamente en un 50\%, registrado en $87 \%$ de los productores de la 
comunidad, con superficies de 6 a 10 ha dedicadas al cultivo del maíz por causa de los altos costos de producción y el poco margen de ganancia actual del cultivo de maíz, que coincide con lo reportado por Guevara-Hernández et al. (2015). Otra causa de la reducción de la superficie sembrada con maíz es el proceso de ganaderización, fundamentado y observado en la comunidad a través del incremento del tamaño de hatos y el número de potreros por productor (Guevara Hernández et al., 2011).

\section{1. 2. Sistema de producción de frijol}

De 1984 a 1990, el sistema de producción de frijol no era de gran interés para los productores debido a las pérdidas de hasta la tercera parte del cultivo por afectaciones en la germinación provocadas por las lluvias abundantes. La producción obtenida se destinaba en su totalidad al autoconsumo y en ocasiones el volumen de grano producido era insuficiente para alimentar a la familia (tabla 2 y figura 2).

TABLA 2

Innovaciones en el sistema de producción de frijol de la comunidad Francisco Villa I (1984-2015)

\begin{tabular}{|c|c|c|c|}
\hline \multirow[b]{2}{*}{ Componente tecnológico } & \multicolumn{3}{|c|}{ Periodo/cambio } \\
\hline & $\begin{array}{l}\text { Periodo I } \\
1984-1990\end{array}$ & $\begin{array}{l}\text { Periodo II } \\
1991-2000\end{array}$ & $\begin{array}{l}\text { Periodo III } \\
2001-2015\end{array}$ \\
\hline Interés local & $\begin{array}{l}\text { No era de importancia } \\
\text { comercial }\end{array}$ & $\begin{array}{l}\text { Nace interés por sembrar } \\
\text { más superficie }\end{array}$ & $\begin{array}{l}\text { Se organizan los } \\
\text { productores para } \\
\text { gestionar apoyos }\end{array}$ \\
\hline Tipo de sistema & Tradicional de bajos insumos & $\begin{array}{l}\text { Tradicional de bajos } \\
\text { insumos }\end{array}$ & $\begin{array}{l}\text { Tradicional de bajos } \\
\text { insumos }\end{array}$ \\
\hline Preparación del suelo & $\begin{array}{l}\text { Eliminación manual de } \\
\text { arvenses }\end{array}$ & $\begin{array}{l}\text { Eliminación de arvenses con } \\
\text { herbicidas }\end{array}$ & $\begin{array}{l}\text { Eliminación de arvenses } \\
\text { con herbicidas }\end{array}$ \\
\hline Tipo de semilla empleada & $\begin{array}{l}\text { Negro huasteco, vaina morada } \\
\text { y vaina blanca }\end{array}$ & Mismo material genético & Mismo material genético \\
\hline Fertilización & Sin aplicación & $\begin{array}{l}\text { Introducción de fertilizantes } \\
\text { químicos }\end{array}$ & $\begin{array}{l}\text { Introducción de } \\
\text { fertilizantes foliares y } \\
\text { compostas }\end{array}$ \\
\hline Control de plagas & Sin control alguno & Instrucción de insecticidas & $\begin{array}{l}\text { Uso de insecticidas } \\
\text { comerciales y extractos } \\
\text { vegetales }\end{array}$ \\
\hline Rendimientos & Bajos & Bajos & Bajos \\
\hline Destino de la producción & Autoconsumo & Autoconsumo & Autoconsumo \\
\hline
\end{tabular}

Fuente: elaboración propia.

Don Nazario "N", habitante de la comunidad, mencionó que antes se cosechaban $1500 \mathrm{~kg}$ de grano en 2 hectáreas (750 kg ha-1), y actualmente el promedio es de $300 \mathrm{~kg} \mathrm{ha}^{-1}$, cuando las "tierras nuevas" aún eran fértiles, lo que demuestra el decremento en los rendimientos por unidad de superficie a lo largo de los años. Esta situación representa baja eficiencia del sistema y pobre adopción de las técnicas modernas de cultivo o el uso de productos químicos desde la implementación, que es un aspecto muy interesante para la innovación en la producción agroecológica. La baja productividad podría sustentarse en dos argumentos: a) el clima y el suelo inadecuados para la producción de frijol y $b$ ) los productores de Francisco Villa I no han encontrado la variedad apropiada para manejarse bajo las condiciones climáticas y de suelo prevalecientes en la comunidad. Ante esto, técnicamente se presenta una oportunidad para la innovación productiva. 


\begin{tabular}{|l|l|l|l|l|l|l|l|l|l|l|l|l|}
\hline Actividad/Mes & Ene & Feb & Mar & Abr & May & Jun & Jul & Ago & Sep & Oct & Nov & Dic \\
\hline Preparación del terreno o limpia & & & & & & & & & & & & \\
\hline Aplicación de herbicidas & & & & & & & & & & & & \\
\hline Siembra & & & & & & & & & & & & \\
\hline Fertilización foliar & & & & & & & & & & & & \\
\hline Control de arvenses & & & & & & & & & & & & \\
\hline Control de plagas & & & & & & & & & & & & \\
\hline Cosecha & & & & & & & & & & \\
\hline
\end{tabular}

FIGURA 2

Calendarización de actividades del sistema de producción de frijol de temporal en la comunidad Francisco Villa I

De los productores encuestados de la comunidad, 83\% siembra frijol con tecnología tradicional para autoconsumo sobre todo porque las lluvias han disminuido y las temperaturas han aumentado, aunque, de acuerdo con versiones locales, estas condiciones climáticas favorecen el cultivo a pesar del bajo uso de tecnologías modernas. Sin embargo, los productores señalan que han aparecido otros problemas, como la plaga conocida como babosa (Sarasinula plebeia, Fisher) y la mosca blanca (Bemisia tabaci, Gennadius).

Los productores practican dos tipos de siembra: el frijol aventurero, que se establece en mayo y el frijol de norte, en agosto. En ambos sistemas la siembra se realiza de forma manual utilizando una macana o espeque. Por causa del alto riesgo de pérdida de cosecha por altas temperaturas, el frijol aventurero se siembra a baja escala, en promedio hasta una cuartilla ${ }^{[5]}$ por productor, básicamente para obtener la simiente que usará en la siembra de frijol de norte que oscila entre 5 y 6 ha también por productor, aunque algunos prefieren sembrar sólo una hectárea. Entre los frijoles locales más usados se encuentran el negro huasteco, el vaina morada y el vaina blanca. El rendimiento aún es bajo pues sólo se obtiene en promedio de los dos sistemas aproximadamente $300 \mathrm{~kg} \mathrm{ha}^{-1}$, con precio de venta entre 14 y $15 \$ \mathrm{~kg}^{-1}$.

Las labores del sistema de producción para el frijol aventurero comienzan con la preparación del suelo en mayo, la maleza se elimina con herbicidas, luego se realiza la quema y las nuevas poblaciones de arvenses se controlan con otra aplicación de herbicida. El sistema de frijol de norte se establece por lo general en las mismas áreas cultivadas con maíz, es decir, en relevo. La siembra de frijol se realiza después de la segunda limpia y segunda fertilización del maíz.

La aplicación de fertilizantes foliares es similar en ambos periodos de siembra y se realiza principalmente en junio (sistema frijol aventurero) y agosto (sistema frijol de norte), con productos como el Growgreen ${ }^{\circledR}$, un fertilizante foliar comercializado en la región.

La cosecha se lleva a cabo cuando las plantas comienzan a secarse, las cuales se extraen completas y se exponen después al sol para culminar su deshidratación y separar la semilla de las vainas. Esta actividad se hace en julio para el frijol aventurero, mientras que el frijol de norte se espera hasta octubre o noviembre. Es decir, el ciclo de producción de frijol en la comunidad es de tres meses.

Las innovaciones en el sistema de producción de frijol han sido más lentas que en el sistema maíz, ya que el frijol está orientado nada más al autoconsumo; no obstante, en los últimos años se observa un interés local por producir suficiente para vender, de ahí que algunos productores han mejorado técnicas de manejo de plagas y de fertilización, así como su forma de organización para gestionar subsidios para el cultivo de frijol. Sin duda, existe una puerta de entrada para la innovación.

\section{1. 3. Sistema de producción ganadero}

La ganadería bovina de doble propósito en la comunidad Francisco Villa I inició en 1985 con la introducción de la raza cebú (Bos primigenius indicus, antes Bos taurus indicus). Posteriormente se incorporó el ganado suizo 
(Bos taurus alpinus), que permitió a los productores realizar sus propios cruzamientos entre ambas razas con el objetivo de mejorar la producción de leche. A partir de 1995 se introducen otras razas como el suizo americano (Bos taurus), lo que propició la mejora genética a partir de la entrada de nuevos sementales de dicha raza (tabla 3 y la figura 3 ).

TABLA 3

Innovaciones en el sistema de producción ganadería bovina en la comunidad Francisco Villa I (1984-2015)

\begin{tabular}{|c|c|c|c|}
\hline \multirow{2}{*}{$\begin{array}{l}\text { Componente } \\
\text { tecnológico }\end{array}$} & \multicolumn{3}{|c|}{ Periodo/cambio } \\
\hline & $\begin{array}{l}\text { Periodo I } \\
1984-1990\end{array}$ & $\begin{array}{l}\text { Periodo II } \\
1991-2000\end{array}$ & $\begin{array}{l}\text { Periodo III } \\
2001-2015\end{array}$ \\
\hline Inicio de la ganadería & $\begin{array}{l}\text { En } 1985 \text { es introducido el } \\
\text { ganado de la raza cebú }\end{array}$ & El sistema está en transición & El sistema está fortalecido \\
\hline Cambios de razas & $\begin{array}{l}\text { En } 1986 \text { es introducida la } \\
\text { raza cebú-suizo americano }\end{array}$ & $\begin{array}{l}\text { En } 1995 \text { es introducida } \\
\text { sangre más pura de la raza } \\
\text { suizo americano (raza } \\
\text { lechera) }\end{array}$ & $\begin{array}{l}\text { Se mantiene la mejora } \\
\text { local de razas con material } \\
\text { genético existente en la } \\
\text { región }\end{array}$ \\
\hline $\begin{array}{l}\text { Mejoramiento en la } \\
\text { alimentación }\end{array}$ & $\begin{array}{l}\text { Los animales eran } \\
\text { alimentados en potreros }\end{array}$ & $\begin{array}{l}\text { En } 1995 \text { mejora la } \\
\text { alimentación con fuentes } \\
\text { externas de insumos } \\
\text { (pollinaza, melaza, maíz } \\
\text { molido, etcétera) }\end{array}$ & $\begin{array}{l}\text { Se busca la mejora de } \\
\text { alimentación con recursos } \\
\text { locales (árboles forrajeros, } \\
\text { silos de maíz y pasto, pastos } \\
\text { de corte) }\end{array}$ \\
\hline Vacunas & No se vacunaba & $\begin{array}{l}\text { En } 1998 \text { con la muerte de } \\
\text { ganado por derrengue se } \\
\text { empiezan a aplicar vacunas }\end{array}$ & $\begin{array}{l}\text { Manejo integrado de la } \\
\text { sanidad. Contratación de } \\
\text { médicos veterinarios }\end{array}$ \\
\hline Capacitación & $\begin{array}{l}\text { Ninguna capacitación } \\
\text { recibida }\end{array}$ & $\begin{array}{l}\text { Reciben cursos de manejo } \\
\text { sanitario }\end{array}$ & $\begin{array}{l}\text { Reciben cursos de manejo } \\
\text { de sistemas silvopastoriles y } \\
\text { ganadería semiestabulada }\end{array}$ \\
\hline $\begin{array}{l}\text { Organización } \\
\text { productores }\end{array}$ & Ningún tipo de organización & Ningún tipo de organización & $\begin{array}{l}\text { Se forma un grupo local para } \\
\text { gestión de créditos ganaderos }\end{array}$ \\
\hline
\end{tabular}

Fuente: elaboración propia.

\begin{tabular}{|l|l|l|l|l|l|l|l|l|l|l|l|l|}
\hline Actividad/Mes & Ene & Feb & Mar & Abr & May & Jun & Jul & Ago & Sep & Oct & Nov & Dic \\
\hline Pica de potreros & & & & & & & & & & & & \\
\hline Compostura de alambres & & & & & & & & & & & & \\
\hline Ordeño & & & & & & & & & & & & \\
\hline Corte y molida de pasto & & & & & & & & & & & & \\
\hline Ensilado de pasto o maíz & & & & & & & & & & & & \\
\hline Vacunación & & & & & & & & & & & & \\
\hline Desparasitación & & & & & & & & & & & & \\
\hline Baños garrapaticidas & & & & & & & & & & & & \\
\hline
\end{tabular}

FIGURA 3

Calendarización de actividades del sistema de producción de ganadería bovina en la comunidad Francisco Villa I 
La muerte de algunos bovinos ocurrida a partir de 1998, a causa de la rabia o el derrengue, demandó el aprendizaje de conocimientos nuevos tales como la aplicación de vacunas para reducir la incidencia de estas enfermedades, lo cual se convirtió en un nuevo elemento del sistema de manejo pecuario y al mismo tiempo emergió como una necesidad; a la postre se incorporó como un componente innovador del manejo de la sanidad de sus animales en un contexto productivo específico de comunidades rurales, dentro o aledañas a áreas naturales protegidas.

El manejo de la salud animal en la comunidad ha evolucionado, de tal forma que en la actualidad se realiza a partir de dos vías preventivas fundamentales: a) la desparasitación y $b$ ) la vacunación, tanto para controlar la presencia de la rabia como para el suministro de vitaminas y contar con animales resistentes a dichos padecimientos. La vacuna se aplica durante los meses de sequía (marzo a mayo), mientras que la desparasitación en el primero y tercer trimestres del año.

La manera como se transmite la información para el control de las enfermedades del ganado es por tradición oral y son los ranchos, casas ejidales y tiendas de productos veterinarios los principales escenarios de intercambio de experiencias. Este es un claro ejemplo de que la innovación es un proceso de aprendizaje social y colectivo, como lo señalan Gómez et al. (2010), a través del cual se intercambia información variada que puede ser objeto de modificaciones, producto de las demandas y necesidades de los propios productores.

El manejo de los potreros, que se realiza entre marzo yjunio, consiste en la eliminación de arbustos, la construcción y la reparación de divisiones para mantener en buen estado los cercos y evitar la fuga de los animales. El cercado en ocasiones se realiza en junio, dado que los suelos están "blandos” por la humedad, la cual se aprovecha para plantar algunas especies forrajeras locales como guash (Leucaena leucocephala (Lam.) de Wit), mata ratón (Gliricidia sepium (Jacq.) Kunth ex Walp) y caulote (Guazuma ulmifolia Lam.) que funcionan a la vez como cercos vivos. Durante todo el año las vacas son ordeñadas de forma manual o mecánica, aunque aún prevalece la primera.

Actualmente, los productores distribuyen sus actividades en diferentes periodos; por ejemplo, el corte y la molienda del pasto lo realizan de abril a mayo. En un inicio el pasto molido se mezclaba con pollinaza, pero eliminaron esa práctica con el argumento de que la leche adquiría el olor característico de ese subproducto avícola. Luego se logró la inclusión de nuevos componentes al sistema, como la alimentación compuesta de pollinaza, melaza, maíz molido y sales minerales con lo que se logró, según los productores, un balance nutritivo. A partir del 2000 se implementó el molinado de los pastos y hoy día se han incorporado nuevas técnicas como el ensilado de pastos y maíz, así como el establecimiento de sistemas silvopastoriles con árboles forrajeros locales. Los cambios señalados dieron forma y origen a un sistema de producción ganadero localmente validado a partir de las innovaciones implementadas y con mejores beneficios para la producción, tanto de carne como de leche, pero bajo un sistema de manejo específico de ganadería semiextensiva.

Los cambios realizados, algunos de los cuales prevalecen en la actualidad, radican en el manejo integral de los recursos naturales para fortalecer el sistema de producción local. Entre las innovaciones más recientes se encuentran la implementación de sistemas silvopastoriles, el uso de silos forrajeros a partir de pastos introducidos y locales o maíz, la contratación de un médico veterinario que apoya el manejo zoosanitario y la gestión de créditos bancarios o gubernamentales para incrementar el tamaño de los hatos ganaderos o mejorar la genética animal, entre otros. La necesidad individual se generalizó para todos los ganaderos locales, lo que significa que una carencia se puede convertir en una oportunidad de innovación social y productiva.

Por tanto, es evidente que una característica emergente de los sistemas locales de innovación, en el que los productores incorporan nuevos elementos a sus sistemas ya establecidos y lo modifican cuando no les son favorables, resulta en un típico experimento de ensayo-error (Gómez et al., 2012; Nahed et al., 2010).

\section{INNOVACIÓN EN LOS SISTEMAS DE PRODUCCIÓN AGROPECUARIA}

Las evidencias previas dan cuenta de tendencias en la evolución e innovación desarrolladas en los tres sistemas de producción analizados. Desde la perspectiva histórica, la comunidad Francisco Villa I, fundada en 1984, 
desarrolla las primeras actividades agrícolas como un sistema roza, tumba y quema (R-T-Q) para abrir campos al cultivo en la porción que en un inicio era la selva baja. La comunidad inicia actividades productivas el mismo año de su fundación con el establecimiento de los sistemas agrícolas, maíz y frijol principalmente; un año después, introdujo la ganadería con la raza cebú. De esta forma, inicia un proceso de cambio, tanto social como económico y productivo, sobre la base de los recursos naturales de la comunidad.

Con base en las percepciones de los productores en la dinámica de cambios generados a lo largo de la historia de la comunidad, de manera general, el sistema de producción ganadero supera al maíz y al frijol en cuanto indicadores de incremento de los ingresos, mejoramiento de la producción, situación personal debido a los cambios y funcionalidad de las innovaciones (gráfica 1).

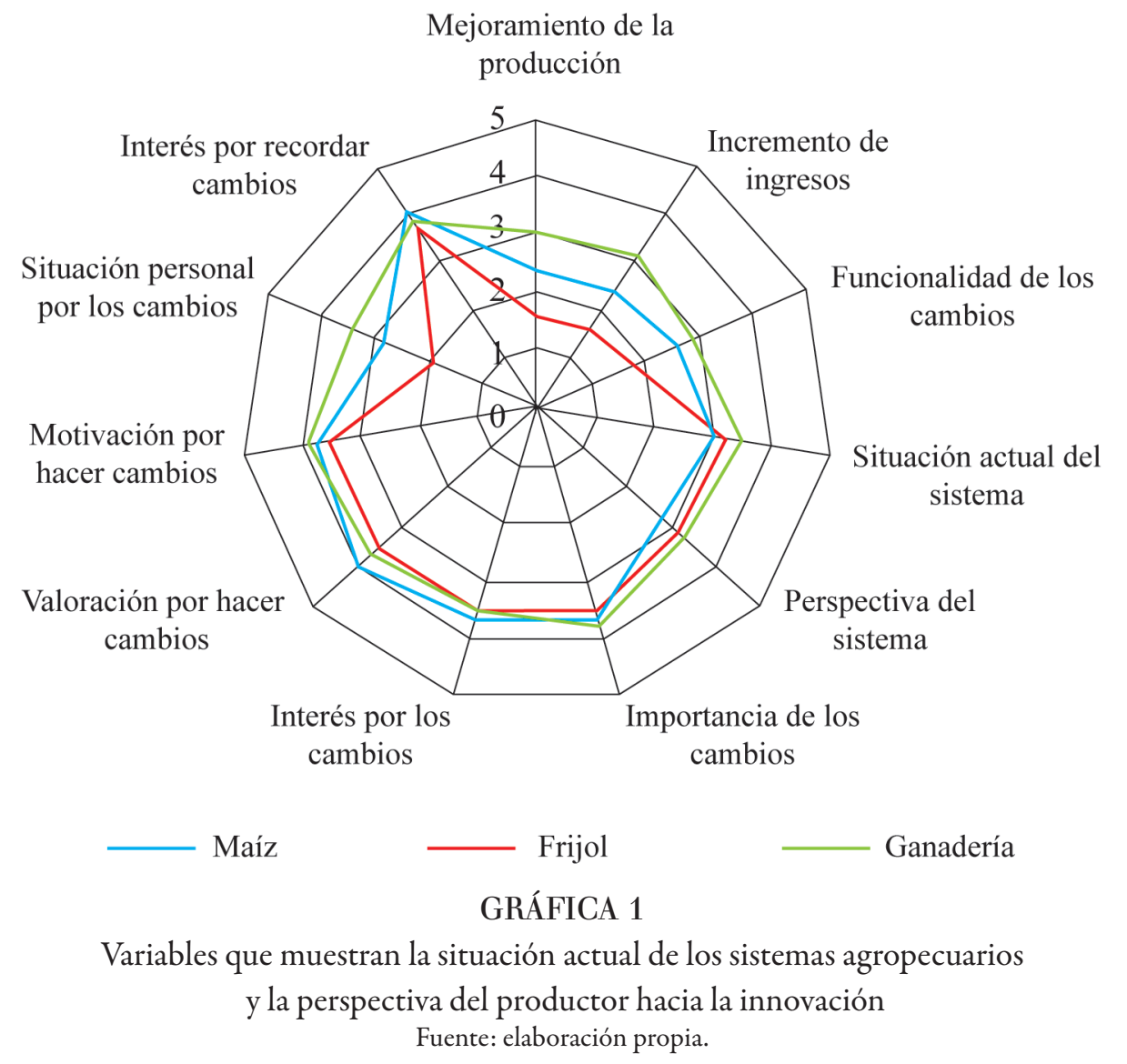

Los resultados del estudio de los tres sistemas de producción agropecuaria en Francisco Villa I demuestran que la funcionalidad de los cambios, a manera de innovaciones, es lo que determina su forma y situación. Entre los componentes mencionados con mayor frecuencia están los productivos (mayor producción de biomasa) y los económicos (más ingresos). También se detectó que en el sistema ganadero existe proporcionalidad entre el mejoramiento de producción y calidad del producto con el incremento de los ingresos y, en consecuencia, la satisfacción del productor. Este resultado coincide con Johnson y Andersen (2012) y Salazar y Rosabal (2007) en cuanto que la innovación de los sistemas es una construcción de fuerzas donde se articulan procesos de desarrollo, siendo éste un factor determinante del cambio económico y bienestar social.

En este sentido, Ruíz (2006) y Sol et al. (2013) apuntan que un sistema productivo es abierto, dinámico y social como consecuencia de interacciones de agentes, como el entorno (ambiente) y el contexto socioeconómico. Se considera que la innovación debe generarse con los actores existentes, por lo que es necesario innovar de manera 
constante (desde adentro), probar experiencias nuevas (apropiación y adopción de innovaciones) tal como lo mencionan Alexander y Martin (2013) y Rodríguez y Alvarado (2008), por lo que se agilizaría la dinámica de la innovación en los sistemas.

Por otra parte, se infiere que el interés de los productores por realizar cambios, así como la importancia que tienen éstos entre sus diversas prioridades, es similar en todos los sistemas. Por ejemplo, en la ganadería bovina, el productor Aurelio "X" menciona: "Es necesario realizar cambios, mucho mejores cambios que ayuden a producir más, como mejorar el ganado y una buena alimentación para éstos”. Lo anterior se explica por la intención de mejorar sus sistemas en todos los componentes. Así también, se observa que las ventajas económicas confieren mayor preferencia al sistema de producción de la ganadería bovina por encima de los de maíz y frijol. Si bien las diferencias en indicadores económicos y productivos son significativas para estos sistemas, el interés, la importancia y la motivación por los cambios muestran un comportamiento análogo en los tres sistemas, lo que define una evaluación similar en cuanto a las perspectivas de todos en dicha comunidad.

Entonces, es pertinente aprovechar estas oportunidades descubiertas y vistas como demandas clave para la innovación en sistemas productivos tan relevantes para las familias de la comunidad Francisco Villa I. Estas oportunidades pueden convertirse en notables "puntos de entrada" para generar procesos de innovación local y el perfeccionamiento técnico de dichos sistemas.

No obstante, se declara que los sistemas de frijol y maíz continúan siendo importantes para la vida de las familias debido a que producen satisfactores primarios directos como el autoabasto en la alimentación familiar. Por ello, resultaría primordial mejorar la articulación con los productores, ya que se requiere estimular y apoyar los cambios internos a través del acompañamiento por parte de los agentes externos como fuente de conocimiento científico e información y así un nuevo conocimiento, nutrido también por los intereses personales y colectivos, vinculados con los aspectos cognitivos trasmitidos de generación en generación para lograr la soberanía y seguridad alimentaria local.

\section{Conclusiones}

Teniendo en cuenta que la innovación constituye un proceso social complejo, creativo y recreativo para conquistar mayor eficiencia y eficacia en los sistemas productivos en beneficio de sus protagonistas, la comunidad y la sociedad en general, derivado del presente estudio, se concluye que los sistemas frijol, maíz y ganadería bovina de doble propósito de la comunidad Francisco Villa I pueden emprender procesos de innovación local si se tiene en cuenta que:

a) Los cambios implementados en los sistemas de producción estudiados históricamente han logrado una adaptabilidad y resiliencia acorde con las condiciones internas y externas cambiantes del contexto.

b) Para la evolución de los sistemas de producción se ha requerido que los productores interactúen de manera intrínseca con factores de riesgo abióticos (lluvias y temperaturas) y bióticos (plagas y enfermedades), así como hacer frente a los cambios en las políticas y leyes relacionadas con el desarrollo y que ciertamente rigen el manejo de los recursos naturales en áreas naturales protegidas, pero que al final les ha permitido obtener resultados favorables en términos de producción y satisfacción familiar.

c) Los conocimientos adquiridos por parte de los productores, tanto de manera empírica como por la interacción con agentes externos, son puestos en práctica como parte de las estrategias de sobrevivencia individual y de desarrollo local de los sistemas productivos. Esto ha permitido que dichos sistemas evolucionen a través del tiempo como consecuencia de la búsqueda de soluciones innovadoras a los problemas cotidianos, y en sus formas de percibir el mundo productivo desde su comunidad. No obstante, la disposición local por aprender y poner en práctica ideas y conocimientos nuevos se demuestra en el interés de los productores en innovar e incluir prácticas novedosas en sus sistemas agropecuarios. 
d) Los productores han sido capaces de innovar, adaptar o adoptar conocimientos y técnicas por dos vías. Una es de forma pragmática a través del ensayo y error, reflejada en su esfuerzo individual y condicionado por la interacción con el medio natural y el medio social, es decir, mediante sus relaciones sociales de producción. La otra vía es por medio del contacto con agentes externos. Esta capacidad individual y colectiva es evidente por los cambios realizados en los sistemas productivos a través del tiempo, en la forma de adoptar las innovaciones y en la socialización de los cambios.

\section{Análisis PRospectivo}

Los cambios en los sistemas de producción pueden ser considerados como "puntos de entrada" para el desarrollo de procesos de innovación agropecuaria a partir de plataformas locales, donde factores internos y externos se integren a la dinámica comunitaria del cambio funcional de dichos sistemas. Es decir, los esfuerzos realizados por las ONG y universidades que confluyen en el área natural protegida aledaña y la comunidad misma representan una oportunidad para actores, tanto comunitarios como externos, para trabajar conjuntamente en procesos de innovación local productiva y desarrollo comunitario. Este tipo de estudios permite descubrir escenarios potenciales de acción, pero también otros campos de investigación como la aplicada o adaptativa y participativa con los productores agropecuarios.

Entre las posibles estrategias de desarrollo local estarían el diseño y operación de un currículo temático para la capacitación de los productores tendiente a fortalecer las capacidades locales para la innovación de los sistemas productivos mediante la experimentación campesina, las escuelas de campo para agricultores u otro enfoque metodológico localmente validado, conforme a cada sistema de producción vigente, del cual depende la dinámica socioproductiva y cultural de la comunidad.

La prospectiva expuesta radica en profundizar en este tipo de estudios para identificar entradas o ventanas para la innovación local mediante el análisis cuantitativo de componentes energéticos y económicos de los sistemas de producción a través del balance de entradas y salidas y finalmente para diseñar un manejo más eficiente de los agroecosistemas.

\section{Agradecimientos}

Este artículo se elaboró como parte de los resultados del proyecto de investigación: Caracterización socioagronómica de maices locales con potencial de uso múltiple en la Frailesca, Chiapas, financiado por la SEPCONACYT en su convocatoria CB2015 y cuyo responsable técnico es el primer autor. Por lo anterior, se agradece al personal del CONACYT, del COCYTECH y de MasAgro-CIMMYT, así como a los productores, estudiantes e investigadores con quienes se trabaja y colabora en la actualidad.

\section{REFERENCIAS}

Alexander, A. T., \& Martin, D. P. (2013). Intermediaries for open innovation: a competence-based comparison of knowledge transfer offices practices. Technological Forecasting and Social Change, 80(1), 38-49.

Almekinders, C. y Guevara, F. (2008). El entorno de FP y la vida de agricultores: estudio de la línea de base y los cambios ocurridos entre 2000-2008 en los proyectos de Fitomejoramiento participativo en Meso-América. Reporte técnico presentado a CIPRES sobre la evaluación realizada al programa mesoamericano de fitomejoramiento participativo. Managua, Nicaragua. 
Almekinders, C., Bukema, L., \& Tromp, C. (2009). Research in action: theories and practices for innovation and social change. Mansholt Publication Series Vol. 6. The Netherlands: Wageningen Academic Publishers.

Arias, G. M., Labrada, C., Hernández, R., Romero, S. L., Vilariño, C. M., Rosabal, Y., González, T., Díaz, L., Forteza, S. A., Perón, E., Torralba, A., Permuy, N., Serrano, R. M., Leyva, M., Batista, D., Jocik, G., Martínez, B. y Peña, M. (2008). Estudios sobre desarrollo local, innovación social y género. La Habana: Editorial Academia.

Arocena, R., \& Sutz, J. (2000). Looking at national systems of innovation from the South. Industry and Innovation, $7(1), 55-75$

Bellón, M. R. (2002). Métodos de investigación participativa para evaluar la tecnología. Manual para científicos que trabajan con agricultores. D. F. CIMMYT.

Corona, L. y Hernández, R. (2001). Innovación tecnológica y medio ambiente. México: Plaza y Valdés Editores.

Giddens, A. (1982). Sociology: a brief but critical introduction. UK: The Macmillan Press Ltd.

Gómez, H., Guevara, F., Hernández, M. H., Nahed, J., Rodríguez, L., \& Pinto, R. (2012). Analysis of cattle raising and institutional perspectives regarding collective action in the "El Ocote" Biosphere Reserve, Chiapas, Mexico. Journal of Animal and Veterinary Advances, 11(6), 831-840.

Gómez, H., Pinto, R., Guevara, F., Medina, F. J., Nahed, J., Ruiz, B. y Mendoza, P. (2010). Innovación local de sistemas silvopastoriles: contribución a la reconversión productiva de áreas ganaderas. Tuxtla Gutiérrez: Universidad Autónoma de Chiapas.

Guevara-Hernández, F., Rodríguez-Larramendi, L. A., Hernández-Ramos, M. A., Fonseca-Flores, M. A., Pinto Ruiz, R. y Reyes-Muro, L. (2015). Eficiencia energética y económica del cultivo de maíz en la zona de amortiguamiento de la Reserva de la Biosfera "La Sepultura”, Chiapas, México. Revista Mexicana de Ciencias Agrícolas, 6(8), 1929-1941.

Guevara-Hernández, F., Pinto R. R., Rodríguez, L. L. A., Gómez, C. H., Ortiz, P. R., Ibrahim, M. y Georgina Cruz. (2011). Local perceptions of degradation in rangelands from a livestock farming community in Chiapas, Mexico. Cuban Journal of Agricultural Science, 45(3), 311-319.

Guevara, F. y Rodríguez, L. (2011). Innovación y desarrollo rural: reflexiones y experiencias desde el contexto cubano. Bayamo: Editorial Jorge Dimitrov.

Guevara, F., Martínez, B., Hernández, A., Pinto, R., \& Ovando, J. (2009). La investigación para el desarrollo rural: ¿qué es la investigación-Acción? CLIA Granma. Boletín del Centro Local de Innovación Agropecuaria, 3(2), 1-2.

Guevara Hernández, F., Hernández López, A., Pinto Ruiz, R. y Ovando Cruz, J. (2008). La investigación para el desarrollo rural: ¿Por qué un proceso continuo? CLIA Granma. Boletin del Centro Local de Innovación Agropecuaria, 2(2), 1-2.

Guevara, F. (2007). ¿Y después qué? Action-research and ethnography on governance, actors and development in Southern México (tesis doctoral). The Netherlands: Wageningen University.

Hernández, E. (1980). Agricultura tradicional y desarrollo. Revista de Geografía Agrícola, 1, 419-422.

Johnson, B., \& Andersen A. D. (2012). Learning, innovation and inclusive development. New perspectives on economic development strategy and development aid. Denmark: Aalborg University Press.

Keune, L. y Boog, B. (2000). Investigación acción ejemplar: conceptos y aplicaciones. San José: Editorial DEI.

Knickel, K., Brunori, G., Rand, S., \& Jet, P. (2009). Towards a better conceptual framework for innovation processes in agriculture and rural development: From linear model to systemic approaches. Journal of Agriculture and Extension, 15(2), 31-146. 
Lundvall, B. A., \& Johnson, B. (1994). The learning economy. Journal of Industry Studies, 1(2), 23- 42

Méndez Gutiérrez del Valle, R. (2010). Estrategias de innovación industrial y desarrollo económico en las ciudades intermedias de España. Bilbao: Fundación BBVA.

Mosse, D. (2005). Cultivating development: An ethnography of aid policy and practice. UK: Pluto Press.

Muñoz, M., Rendón, R., Aguilar, J., García, J. G. y Altamirano, J. R. (2004). Redes de innovación: un acercamiento a su identificación, análisis y gestión para el desarrollo rural. Fundación Produce Michoacán, A. C.Universidad Autónoma Chapingo.

Nahed, J., Gómez, H., Pinto, R., Guevara, F., Medina, F., Ibrahim, M., \& Grande, D. (2010). Research and development of silvopastoral systems in a village in the buffer zone of the El Ocote Biosphere Reserve, Chiapas, Mexico. Research Journal of Biological Sciences, 5(7), 499-507.

Partida, R. (1996). Las tecnópolis del mundo: la formación de los complejos industriales del siglo XXI. Espiral: Estudios sobre Estado y Sociedad, 26, 209-225

Represas, J. (1962). Ciencia y tecnología de la industria. Folia Universitaria. Guadalajara: Universidad Autónoma de Guadalajara.

Rist, S., Chiddambaranathan, M., Escobar, E., \& Wiesmann, U. (2006). It was hard to come to mutual understanding. The multidimensionality of social learning processes concerned with sustainable natural resource use in India, Africa and Latin America. Systemic Practice and Action Research, 19(3), 219-237

Rhodes, R. y Bebbington, A. (1988). Farmers who experiment: An untapped resource for agricultural development. Lima: Centro Internacional de la Papa (CIP).

Rodríguez, A. y Alvarado, H. (2008). Claves de la innovación social en América Latina y el Caribe. Santiago de Chile. Chile. Impreso en Naciones Unidas.

Rogers, E. (1983). Diffusion of innovations. New York: Free Press.

Röling, N. (1996). Towards an interactive agricultural science. Journal of Agricultural Education and Extension, $2(4), 35-48$.

Ruíz, G. (2006). La innovación: un proceso social e interactivo basado en el conocimiento. Colombia: Instituto Tecnológico Metropolitano.

Salazar, L. y Rosabal, Y. (2007). Procesos de innovación rural: una mirada al desarrollo rural desde la reflexión y experiencia de América Latina. Barquisimeto. Venezuela: Editorial Digesa Lara, S. A.

Salomón, M. y Engel, P. (1999) Facilitando la innovación para el desarrollo. Una caja de recursos para la ERSICA. Chile: RIMISP.

Santiago, B. A. (1985). Investigación, invención e innovación. México: Universidad Nacional Autónoma de México.

Scoones, I., \& Thompson, J. (1994). Beyond farmers first: rural people's knowledge, agricultural research and extension practice. London: Intermediate Technology Publications.

Selener, D. (1997). Participatory action research and social change. The Cornell New York: Cornell University. Ithaca, N.Y.

Sol, J., Beers, P. J., \& Wals, A. E. J. (2013). Social learning in regional innovation networks: trust, commitment and reframing as emergent properties of interaction. Journal of Cleaner Production, 49, 35-43

Toledo, V. M., Carabias, J., Mapes, C. y Toledo, C. (1985). Ecología y autosuficiencia alimentaria. México: Editorial Siglo XXI.

Uphoff, N. (1986). Local institutional development: an analytical source book with cases. London: Kumarian Press. 
Waitley, D., \& Tucker, R. B. (1989). Winning the innovation game. New York: Berkley Books.

Wenger, E., McDermott, R., \& Snyder, W. (2002). Cultivating communities of practice: a guide to managing knowledge. Cambridge, M. A.: Harvard Business Press.

Young, H. (2009). Innovation diffusion in heterogeneous populations: contagion, social influence, and social learning. The American Economic Review, 99(5), 1899-1924.

\section{Notas}

[1] "Gente con derecho a uso-compra y venta de la tierra. Poblador: gente con derecho a vivir en la comunidad pero sin tierra, pueden usar la tierra bajo préstamo y son ejidatarios una vez que ganan el derecho de serlo [...] ese derecho se gana de muchas maneras, entre ellas, ofreciendo servicio social a la comunidad durante un tiempo o comprar el derecho a alguien que ya no quiera ser ejidatario o que haya emigrado" (Guevara, 2007: 24, 62).

[2] Práctica agrícola tradicional conocida como roza-tumba-quema (R-T-Q), la cual consiste en la eliminación de arbustos y quema de todo este material para posteriormente dejar listo el campo para la siembra.

[3] Instrumento rudimentario que consiste en una vara, rama o tallo puntiagudo en su extremo usado para abrir el orificio en el suelo donde se depositará el grano de frijol o maíz.

[4] Estiércol de ganado.

[5] Medida usada por el productor que equivale aproximadamente a 3-5 $\mathrm{kg}$ de semilla.

\section{BY-NC-ND}

